ALPHA N² 27 Diciembre 2008 (77-92)

ISSN 0716-4254

http://alpha.ulagos.cl

\title{
ESTRATEGIAS DE CORTESÍA VERBAL EN INTERACCIONES ARGUMENTATIVAS OCURRIDAS EN CONTEXTO ESCOLAR ${ }^{1}$
}

Verbal Strategies of courtesy in argumentative interactions ocurring in schoolcontexts

Juan Salazar Parra*

Resumen

Este artículo presenta las fases de la argumentación oral en la interacción y las estrategias de cortesía que regulan dicha interacción en una situación de aula guiada y en otra espontánea (Cros, 2003). Asimismo, se exponen los conceptos clave del estudio de la argumentación, de sus fases de desarrollo y de las estrategias de cortesía que, según Cros, se emplean en una discusión oral en la sala de clases. En ambas situaciones analizadas, los alumnos presentan problemáticas generales, como concesiones, empleo de estrategias de cooperación, desarrollo de una extensa fase de argumentación planteando diversas tesis y argumentos, utilizando estrategias de cortesía que les permiten minimizar los actos amenazadores que evitan coaccionar al interlocutor. Además, se aprecia que sólo en la interacción guiada se puede hallar una fase de cierre que termine con la discusión inicial.

Palabras clave: Interacción argumentativa oral, estrategias de cortesía, fases de la argumentación.

\begin{abstract}
Oral argumentation supposes different domains, like strategies of courtesy, that make the interactive relationships easier (attenuating imposition and promoting agreement). This is why we pretend to detect the argumentation stages in the interaction and the courtesy strategies that regulate a guided situation and a spontaneous one. Also, we present key concepts about argumentation study, stages and courtesy strategies that, following Cros (2003), must be used in an oral discussion in the classroom. Ultimately, we can say that students present general problems as concessions and use cooperation strategies in both situations, they develop an extended arguing stage, planning thesis and arguments, utilizing courtesy strategies that minimize the threatening acts and avoid coercing the interlocutor. Finally, just in guided interaction we find the closing stage that finishes the initial disagreement.
\end{abstract}

Key words: Oral argumentative interaction, courtesy strategies, argumentation stages.

1 Estudio realizado en el marco del proyecto FONDECYT 1060439: "La competencia argumentativa oral en el aula: Un estudio exploratorio con estudiantes de enseñanza media”. 
Juan Salazar Parra

\section{INTRODUCCIÓN}

La habilidad para argumentar oralmente involucra, siguiendo a Marinkovich (2007a), el dominio de ciertas estrategias verbales que harán posible el desarrollo de las ideas o creencias en conflicto. Se trata, así, de un dominio relativo a un entorno comunicativo específico: según el género de la argumentación involucrado, el contexto institucional, la espontaneidad de la situación, etc. La competencia argumentativa no se alcanza de modo obligatorio. Su aprendizaje depende tanto de su importancia en los ámbitos educativos como del lugar que ocupan en una sociedad las prácticas de argumentar, persuadir y convencer por medios verbales. En este sentido, la argumentación puede concebirse como un instrumento cultural que le permite a los sujetos adquirir un desempeño verbal coherente con las funciones ciudadanas (cognitivas y socioculturales) que los diversos contextos demanden, dando "oportunidad para la elección de un punto de vista personal respecto de algún tema polémico" y apreciando "el aporte de estas actitudes para la formación personal y la convivencia democrática” (MINEDUC, 2004).

La realidad descrita hace necesario abordar el estudio de la argumentación oral o, más bien, de la interacción argumentativa en la sala de clases, para así conocer y evaluar qué sucede en ese contexto cuando los actores toman una posición en una discusión. Este diagnóstico permitirá tomar decisiones que se relacionan tanto con los supuestos teóricos como con las metodologías que mejor respondan a la enseñanza-aprendizaje de la argumentación oral en el aula.

Por lo mismo, el propósito de un estudio como éste — de naturaleza cualitativo-interpretativa - es detectar las fases de la argumentación en la interacción y las estrategias de cortesía que regulan una interacción guiada por el profesor y otra espontánea. Ambas interacciones, en el contexto de la sala de clases, intentan resolver un problema y, para ello, los estudiantes toman distintas posturas acerca de un tópico, sustentándolas con argumentaciones que chocan entre sí.

Para tales efectos se indicarán los conceptos claves de la argumentación y de sus fases y de las estrategias de cortesía que concurren en una discusión oral en el aula (Cros, 2003), tanto como los objetivos, el corpus a analizar y la metodología de trabajo, conjuntamente con los datos, una breve discusión de ellos y algunos comentarios.

\section{MARCO DE REFERENCIA}

La argumentación oral corresponde a una actividad que supone el empleo eficaz de diversos dominios, entre los que se hallan estrategias que 
tienden a facilitar las relaciones en la interacción, atenuando la imposición y favoreciendo el acuerdo. De ahí que, como objeto de estudio, se ha constituido en una temática recurrente. Prueba de lo anterior son las líneas teóricas que han surgido en torno a esta actuación verbal y las que pueden servir de apoyo para la descripción de la argumentación oral en el aula. La neorretórica de Perelman y Olbrechts-Tyteca (1958) se orienta al estudio de la argumentación como teoría del razonamiento práctico sustentado en la experiencia, los valores y las creencias. La nueva retórica subraya el carácter dialógico de los procedimientos argumentativos y establece las categorías de argumentos posibles para lograr la adhesión de la audiencia. El modelo de Toulmin (1958) mantiene como punto de referencia el razonamiento lógico, enfocando la estructura de los argumentos y los datos que los apoyan. La pragma-dialéctica de van Eemeren y Grootendorst (1992) apunta a identificar los movimientos dialógicos que se realizan en una argumentación - considerada como una combinación de actos de habla- y a asegurar las condiciones para el desarrollo de una argumentación que conduzca al acuerdo. La pragma-dialéctica se centra en un tipo de diálogo argumentativo como es la discusión crítica cuyo planteamiento consiste en un modelo ideal para la interacción dialéctica entre el proponente y el oponente que permite analizar y evaluar los argumentos conforme a su participación en la resolución de una diferencia de opinión (van Eemeren y Grootendorst, 1992).

En este mismo sentido, Baker (1999) plantea que la argumentación vista desde una dimensión dialéctica es un tipo de juego dinámico en que alguno de los sujetos del diálogo aparece como ganador y el otro como perdedor. El análisis de Baker (1999) está basado en la dialéctica formal de Barth y Krabbe (1982) en la que se aprecia un conjunto de concesiones para iniciar el debate y exponer una tesis que luego será replanteada por los participantes que asumen los roles de proponente y oponente. Así, existirían dos tipos básicos de conflictos: "simple", en el cual una tesis se debate y se pone en duda por parte del oponente, y "mixto", en el que cada participante propone una tesis (tesis y contratesis).

Como señala Anna Cros (2003:13-14), la argumentación es “una actividad discursiva orientada hacia la finalidad de influir sobre las creencias, los valores, las actitudes, los conocimientos de los destinatarios, con tal de modificarlos, si es necesario, y ponerlos de acuerdo con los del enunciador". Pero, la finalidad que persigue la argumentación, sobre todo la oral, en una discusión, exige una actitud positiva del enunciador con respecto al destinatario: puede elogiarlo valorando sus conocimientos, sus capacidades de decisión, su honradez o el hecho de compartir los mismos valores. La noción de "face" que elaboró Goffman (1981), o sea, la imagen que se ostenta ante el interlocutor encaja perfectamente con estas consideraciones. Incluso, esta 


\section{Juan Salazar Parra}

misma noción ha influido en los estudios de lo que hoy se denomina "cortesía verbal", entendida como "el conjunto de procedimientos comunicativos que sirven para lograr mejor el propósito de la comunicación. No se trata de adornos o rebuscamientos, sino de estrategias para lograr presentar una imagen favorable de uno mismo y para establecer una relación cómoda con el interlocutor, que permita ganar eficacia o influencia” (Reyes, 1998:359-360).

La “cortesía”, pues, es una de las soluciones posibles frente al problema de la variación en las convenciones y las posibilidades del enunciador, a partir de los mecanismos que regulan el comportamiento verbal en un contexto dado.

Los factores que se tienen en cuenta en la cortesía son la relación vertical (el poder), la relación horizontal (el grado de conocimiento) y la naturaleza de dicho acto de habla (si se trata de un acto que favorece la cortesía o no, si afecta la faz positiva o la negativa de los participantes). Tales factores tienen repercusiones de menor grado en las interacciones donde prima la transmisión de información (principio de cooperación) y mayor énfasis en las que nos regimos por motivos como el mantener el contacto dando una lectura más social o afectiva a las intervenciones (principio de cortesía).

La propuesta de Brown y Levinson (1987) —entre otros- - parte de un concepto de hablante racional (típicamente occidental) cuya faz o imagen pública tiene un aspecto positivo y otro negativo. El primero consiste en la necesidad de ser aprobado por los demás, de ser apreciado e incluido en un grupo, mientras que el segundo es el deseo de que nadie nos imponga nada, de conservar nuestra independencia. La argumentación dependerá, entonces, de la naturaleza del acto (es decir, de su amenaza potencial para alguna de las caras del individuo, si las protege o las evita), del eje vertical y del eje horizontal.

Según la propuesta de Brown y Levinson (1987), la característica principal de las reglas de cortesía, comportamiento o adecuación al contexto, es la racionalidad. En una situación dada, salvaguardamos nuestra faz negativa, individual, pero, también queremos ser apreciados mediante la faz positiva, social (Brown y Levinson, 1987). Todo ello significa minimizar lo descortés (el costo para el oyente) y maximizar lo cortés (el beneficio para el oyente). Así también lo destaca Escandell

Aunque no vamos a ahondar en detalles y escuelas, sí apuntamos que el estudio de la cortesía tiene como punto de partida el concepto de acompañar un acto de habla con una lectura social, cuando el uso del lenguaje tiende a mantener el equilibrio entre las diferentes posiciones que se relacionan en el discurso (1996:135-136). 
La cortesía, a diferencia de los buenos usos y las buenas costumbres (o sea, aquello que normalmente se entiende por cortesía) es una norma social que regula el comportamiento adecuado de sus miembros (un mecanismo para que la agresividad de sus miembros no se vuelva contra ellos mismos).

Ahora bien, si la argumentación oral se desarrolla en el contexto de la sala de clases, género discursivo que se transmite fundamentalmente por la vía oral y que es gestionado — en la mayoría de las veces - por el profesor, una persona experta que interactúa con un grupo de alumnos, personas no expertas o menos expertas (Cros, 2003), es necesario completar este marco de referencia con una serie de definiciones operativas que permitirán analizar en este estudio las interacciones argumentativas orales en el aula.

Las estrategias argumentativas facilitan las relaciones en la interacción (atenuando la imposición y favoreciendo el acuerdo), ya que "marcan y reflejan la relación que se establece entre los interlocutores en un acto comunicativo en los ejes de poder y solidaridad, de distancia y proximidad, de afecto, etc.” (Calsamiglia y Tusón, 1999:162).

La interacción es aquella acción esencialmente comunicativa e interpersonal, lo cual implica que es realizada por un hablante con la intención de producir un efecto, más o menos, cognitivo en el oyente mediante el reconocimiento de este último de la intención del hablante para producir dicho efecto (Grice, 1975). Aunque las acciones comunicativas pueden ser no verbales, en este estudio nos centraremos en las acciones verbales.

Este concepto de interacción argumentativa, más allá de considerarlo congruente con nuestros propósitos — y tal como lo menciona Baker (1999) no se tomará estrictamente de los teóricos anteriormente citados (sea argumentación como texto); como tipo de acto de habla, como forma lógica, remite a una interacción comunicativa verbal en la cual la dimensión dialéctica está presente.

Lo anterior significa que la argumentación debe satisfacer algunas condiciones mínimas: a) fase de apertura, en la que se reconoce el conflicto verbal expresado mediante diferentes posiciones (a favor/en contra; pro/contra) o actitudes (creencia/no creencia) con respecto a los puntos de vista; b) una fase de argumentación, ocurrida luego de la fase de apertura, en la que cada participante debe, al menos, realizar un acto comunicativo o un enunciado que sea congruente globalmente con las posiciones expresadas en la fase de apertura; estos actos comunicativos que se revelan y se producen de acuerdo con las posiciones a favor/pro son denominados "defensas" y aquellos que corresponden a un en contra/contra, se denominan "ataques"; c) una fase de cierre en que los participantes discuten cómo el conflicto verbal podría cerrarse: ¿Quién tiene la razón o quién está equivocado? ¿Quién ganó o perdió? Sin embargo, se ha constatado que los estudiantes dejan el cierre o 


\section{Juan Salazar Parra}

resolución implícitos. Por esta razón, la fase de cierre no es estrictamente esencial para el análisis de interacciones reales.

Las expresiones de cortesía deben formar parte de esta cadena argumentativa, aunque sea interrumpiendo el razonamiento, constituyendo así estrategias óptimas para atraer la disposición de la audiencia en el desarrollo de la discusión. Estas estrategias se sistematizan, según Cros (2003), en aquellas que tienen un carácter antiamenazador o positivo, como el hecho de manifestar un acuerdo, en otras que sirven para atenuar o evitar los enunciados que podrían ser negativos para la imagen del destinatario, como mostrar deferencia, y las que son encubiertas o indirectas, como es el caso de la ironía. Una propuesta de clasificación se esquematiza en la Tabla 1.

\section{TABLA 1. TIPOS DE ESTRATEGIAS DE CORTESÍA Y SU CODIFICACIÓN}

\begin{tabular}{|c|c|c|c|}
\hline & Tipos & Subtipos & Codificación \\
\hline \multirow{7}{*}{ Estrategias de cortesía } & \multirow{3}{*}{ Cortesía positiva } & Terreno común & {$[+] \mathrm{TC}$} \\
\hline & & Cooperatividad & {$[+] \mathrm{COOP}$} \\
\hline & & $\begin{array}{l}\text { Satisfacer los deseos del } \\
\text { otro }\end{array}$ & {$[+]$ SAT } \\
\hline & \multirow[t]{2}{*}{ Cortesía negativa } & No coaccionar & {$[-] \mathrm{NC}$} \\
\hline & & Minimizar amenazas & [-]MIN \\
\hline & \multirow{2}{*}{$\begin{array}{l}\text { Cortesía } \\
\text { encubierta }\end{array}$} & Ironía & {$[*]$ IRO } \\
\hline & & Ambigüedad & [*]AMB \\
\hline
\end{tabular}

Respecto a las tres estrategias de cortesía positiva, las de Terreno Común apelan a la valoración del destinatario, a la pertenencia a la comunidad sociodiscursiva y hacen referencia a los conocimientos comunes; las de Cooperatividad sirven para expresar que los interlocutores se incluyen recíprocamente en la actividad y las que permiten al enunciador Satisfacer los deseos del otro, utilizando la simpatía.

Las estrategias de cortesía negativa han dado lugar a dos subtipos: No Coaccionar, que ofrecen opciones al interlocutor y las que minimizan amenazas, por medio de la deferencia y atenuando los actos directivos.

Por último, Ironía y Ambigüedad son estrategias de cortesía encubierta, es decir, se producen en forma indirecta y, por ello, no se les puede atribuir una única intención comunicativa.

\section{EL ESTUDIO}

Nuestro propósito es describir cómo argumentan oralmente los estudiantes de un tercer año medio de la asignatura Lengua Castellana y 
Comunicación de un establecimiento educacional particular-subvencionado y confesional de la ciudad de Valparaíso cuando desarrollan la Unidad La argumentación. Específicamente, se trata de determinar, por un lado, las fases de la interacción comunicativa oral de tipo argumentativo (apertura, argumentación y cierre) en el aula, que se observan en las intervenciones tanto del profesor como de los estudiantes cuando discuten acerca de un tema polémico - en este caso, El embarazo adolescente- en una interacción guiada por el profesor y, en otra espontánea, se pretende comparar las estrategias de cortesía verbal que regulan ambas interacciones.

Las transcripciones de dos sesiones de Lengua Castellana y Comunicación en las que se debate acerca del embarazo adolescente constituye el corpus de la investigación. Uno de estos registros corresponde a una sesión de 85 intervenciones, en las que participan siete estudiantes, quienes simulan un debate televisivo. Si bien en esta discusión no aparece el profesor como interlocutor, es él quien ha propuesto la actividad y ha entregado las instrucciones de desarrollo. Por lo mismo se trata de una interacción argumentativa oral, "guiada". En la otra sesión se producen 86 intervenciones y en ella participan el profesor y 18 alumnos, de un total de 42. Se trata, aquí, de una actividad "espontánea”, debido a que los alumnos no han sido instruidos en metas ni en desempeños; se realiza una discusión grupal-plenaria y todos los estudiantes tienen la posibilidad de participar en ella.

Metodológicamente, el análisis de las secuencias orales se realizó sobre la base de su trascripción y en ella se marcaron las fases de la interacción argumentativa según la propuesta de Baker (1999), y se numeraron las intervenciones correspondientes (Marinkovich, 2007a, b). Asimismo, en la primera fase se identificaron aquellas intervenciones que correspondían a la tesis, el ataque o la defensa. En la argumentación se destacó el acto de ataque y de defensa, mientras que en el cierre, si se presentaba, se indicó si se refería al ataque o defensa de algún punto de vista. En estos tramos, se identificó con la letra $\mathrm{P}$ al profesor y con la letra $\mathrm{A}$, seguida de un número, al estudiante. $\mathrm{Al}$ respecto se presentan ejemplos demostrativos de cada una de estas sesiones para poder realizar el análisis y emitir los comentarios.

También se detallan las estrategias de cortesía empleadas por los interlocutores en las distintas intervenciones, determinando el tipo de estrategia que se evidenciaba en los enunciados emitidos, según la codificación descrita en la Tabla 1. Cada acto amenazador de la imagen, ya sea positivo o negativo, de los interlocutores fue localizado en la proposición que contenía dichas estrategias.

Finalmente, los resultados de la aplicación de las distintas categorías de análisis se interpretaron cuantitativa y cualitativamente, en el sentido de dar 
cuenta de diferencias entre la calidad de las intervenciones, tanto desde su aporte argumentativo como del uso de las variadas estrategias de cortesía entre una y otra actividad de argumentación.

\section{ANÁLISIS DE SECUENCIAS Y DISCUSIÓN DE DATOS}

Por estos efectos, se presentan ejemplos demostrativos de cada una de las intervenciones seleccionadas para poder realizar el análisis y, luego, discutir la pertinencia de las interpretaciones que se puedan establecer. A su vez, cada interacción (tanto la "espontánea” como la "guiada") fue esquematizada en sus fases de desarrollo.

Las fases de la argumentación en la interacción "espontánea” relativas a la problemática del embarazo adolescente se analizan en consonancia con las tesis planteadas, lo que significa que al surgir una tesis, debieran surgir las fases correspondientes. Se observaron tres tesis o puntos de vista acerca de la problemática en cuestión, las que emergen de una tesis general subyacente que correspondería a "El embarazo adolescente tiene responsables: La futura madre, los jóvenes involucrados, la educación o la sociedad”.

Tal como se ha señalado, a la tesis general se le han atribuido tres tesis alternas. En su fase de apertura, la tesis 1 fue propuesta por el profesor (P): “El embarazo adolescente es una irresponsabilidad o, sea, las niñas que quedan embarazadas jovencitas provocan esta situación porque son irresponsables”. Este punto de vista es cuestionado por el ataque del alumno 1 (A1): "Es una irresponsabilidad de parte de las dos personas”.

En la tesis 2, la etapa de apertura es nuevamente iniciada con la intervención de $\mathrm{P}$, quien plantea: "Si en algún minuto una niña queda embarazada acá en el colegio deja de estudiar para que se dedique exclusivamente al cuidado de su embarazo y el varón, si es alumno del colegio, también se debe ir, para ponerse a trabajar”. Tal opinión responde a la autoridad del ámbito educativo. Esta tesis se retoma en el turno 28 (intervención 16 de A8) donde aparece el punto de vista contrario, expresado en su primera parte como una generalización algo confusa: "Si a un niño no le permiten volver al colegio, o sea, si no le permiten seguir estudiando, o sea, ¿Qué pretenden? ¿Que la mamá sea una ignorante?”.

Por su parte, la tesis 3 es también expuesta por P: "Falta aquí información". Ante este enunciado, A3 responde con un punto de vista opuesto: "La información está”, a lo que P reacciona, señalando mediante una pregunta, en la que se advierte su posición en el conflicto: “¿Está? ¿Totalmente?”.

Consumada la apertura de la tesis 1, sigue una fase de argumentación con enunciados de ataque: "Porque no sólo la mujer tiene que cuidarse sino 
que también el hombre” y “Típico que el hombre piensa no si a mí, que soy muy chico, no me va a pasar" (tales enunciados responden a argumentos del mismo A1 quien defiende su punto vista). A3 interviene apoyando a A1, pero con una restricción: "Obviamente la mayor responsabilidad recae en las dos personas, pero yo creo que es algo un poco más grande... No es que la culpa no la tenga, es como algo como más de formar un criterio".

Por otra parte, en las intervenciones 14, 15, 17, 18 y 19 de la tesis 2, se formulan los distintos argumentos que, por una parte, atacan dicha tesis y, por otra, la apoyan. A modo de ejemplo, se indican algunas de estas intervenciones de ataque

[1] Porque en primer lugar si la mamá decide o no seguir estudiando es decisión de la mamá [2] Se supone que si una alumna sigue estudiando estando embarazada es porque ella tiene el apoyo de la familia [3] Porque no creo que siga estudiando y pagando por ello siendo que no tiene apoyo y no tiene plata

De entre las intervenciones de apoyo para que la niña embarazada se preocupe de ser una buena madre, se señala: "Después va a nacer y va a crecer en las peores condiciones si la mamá no se preocupa desde ya, entonces, yo pienso que ya tiene que ir formando una familia como todos los niños normales".

Contrariamente a lo sucedido con la expresión de la tesis 1 , la tesis 2 genera puntos de vista en la apertura y argumentos de ataque y de defensa en su desarrollo. Incluso, podemos advertir un intento de cierre más acabado. Todo esto permite calificar este tramo como productivo desde el punto de vista estructural y discursivo.

A la fase de apertura de la tesis 3, le siguen — en la fase de desarrollocuatro argumentos a favor y uno en oposición. Un ejemplo que ilustra la postura en contra de A3, argumenta "Pero la que está muchas veces no la usamos bien", mientras que $\mathrm{P}$ esgrime el argumento “¿Es suficiente la información?, lo que en cierta medida prueba su insistencia a favor del problema de la información en el caso del "embarazo adolescente". A la última pregunta de P, A3 replica: "Nunca va a ser suficiente”.

Para efectos de la fase de cierre, en el desarrollo argumentativo de la tesis 1 observamos que se manifiesta una postura en contra y no se expresa una defensa. La postura en contra, en la fase de argumentación propiamente tal, se fundamenta en dos argumentos del mismo alumno y se complementa con la de otro alumno, que hasta cierto punto, podría interpretarse como un cierre. 


\section{Juan Salazar Parra}

Con respecto a la tesis 2, es necesario destacar que si bien al término de las intervenciones el argumento de ataque de A10 propone: "Si la niña está pidiendo de que siga estudiando es porque ella puede”, este argumento podría, también, constituir una contratesis a la tesis original y, de este modo, considerarse como un cierre al conflicto suscitado.

Finalmente, si bien, la tesis 3 no es tan fecunda como la tesis 2, P cierra la discusión de esta tesis con una reformulación de la tesis inicial: "Y eso entonces, tú, de alguna manera responsabilizas también a la sociedad, a la educación”, lo que podría interpretarse como que el embarazo adolescente es de responsabilidad de la sociedad y de la educación.

\section{FASES DE LA ARGUMENTACIÓN EN LA INTERACCIÓN “GUIADA”}

Las fases que se pueden observar en la interacción argumentativa "guiada" se analizan en consonancia con las tesis planteadas, lo que significa que al surgir una tesis debieran surgir las fases correspondientes. Interesa destacar que, a diferencia de la interacción “espontánea”, en este caso, todas las intervenciones guardan relación con la tesis inicial, que corresponde a "El aborto debe legalizarse en Chile” y que ha sido propuesta por A1 en su rol de moderador del debate televisivo.

En su fase de apertura, la tesis inicial (tesis 1) ha sido enunciada como "El aborto debe legalizarse en Chile”, propuesta por el alumno moderador del debate y se presenta con un apoyo inmediato entregado por uno de los alumnos (A2) que interviene en representación de un sociólogo: "El aborto es un acto más humanitario que cualquier otra opción ante un embarazo no deseado”. A esto, le sigue el conflicto o el ataque interpuesto por A3 (en el rol de una religiosa) quien argumenta: "Según la ley de Dios, [el aborto] es un asesinato, por ende, viola los Derechos Humanos [...] el derecho a la vida que posee todo ser humano".

En el transcurso de la discusión surge una nueva subtesis (tesis 2), a raíz de la argumentación de A3 (que es un ataque a la tesis inicial) en la séptima intervención: "Una de las posibles opciones podría ser que los padres den en adopción al hijo para desarrollar una mejor calidad de vida”. Este punto de vista será retomado sólo a partir de la intervención 20, en la que se puede observar una etapa clara de apertura, con un argumento de apoyo: "Existen diferentes hogares que se encargan cien por ciento de cuidar a los niños”, y su respectiva contra-argumentación: "Pero, nadie toma en cuenta que los niños que sobrepasan los cuatro años dentro del hogar y quedan sin ser adoptados, quedan igual sin familia”.

En su fase de argumentación, la tesis 1 presenta argumentos de ataque, del tipo "Porque no se quiere enfrentar la realidad"; "Porque sabía que mi 
pareja estaría conmigo, pensara lo que pensara mi familia”; “El bebé que yo llevo en mi vientre no tiene la culpa de mi actos y los de mi pareja”. La mayor parte de estos argumentos son expresados por la alumna A7 que, además de plantearlos, se encuentra en estado de embarazo real al momento del debate televisivo. Ella misma argumenta, en una de sus intervenciones, una tesis alterna que no genera discusión: "El aborto es un acto de cobardía”. El debate también genera argumentos de apoyo: "La legalización del aborto puede reducir completamente la introducción de abortos clandestinos"; "Las mujeres contarían con el establecimiento de mejor calidad de vida”.

Por otro lado, la tesis 2 presenta una extensa fase de argumentación que sobrelleva casi la totalidad de la actividad "guiada". A favor de la tesis 2 se argumenta que "en los hogares de acogida se trata a los niños como en cualquier hogar o familia", lo que provoca concesiones: "Por ende [...] que no se sobrepasen los límites”. A lo anterior se le oponen los argumentos de A6 y A2: "Es que tú no vas a estar ahí viendo que no ocurra" o "Esas personas que están a cargo de esos niños no cumplen el rol de padres”.

Finalmente, la tesis 2 no presenta un cierre, tal vez, debido a que corresponde a una especificación temática de lo planteado o lo que se pueda extender de la tesis 1 . Sin embargo, esta última, claramente posee la etapa de cierre de la argumentación, pues, por un lado se concede el rechazo a la tesis inicial, ya que el moderador indica: "Chile no está preparado para la legalización de un aborto, ya que no es un país desarrollado” y, por otro, deja abierta la posibilidad de seguir la discusión acerca del tópico: "[Es] un tema que nunca va a tener solución”. En este sentido, a pesar de que esta última intervención de la moderadora es amplia - y un tanto difusa - el cierre en esta interacción "guiada" presenta mayor claridad tanto temática como estructuralmente, con respecto a lo ocurrido con la interacción “espontánea”.

\section{DIFERENCIAS DE EMPLEO DE ESTRATEGIAS DE CORTESÍA EN LAS INTERACCIONES “GUIADA” Y “ESPONTÁNEA”}

Las estrategias de cortesía del profesor regulan la interacción "espontánea”. Se encuentran tanto en el inicio de la clase, a modo de concesiones, como durante el desarrollo de las fases argumentativas. Inicialmente, predominan las estrategias de Cooperación y, en menor cantidad, las que apelan al Terreno Común; ambas son de imagen positiva, con una escasa incidencia de estrategias de la imagen negativa, como la Minimización de Amenazas y el No Coaccionar, en ejemplos como: "Porque en un principio pensamos en el embarazo adolescente"; "Fueron opciones de curso", o "Entonces tenemos al final tres temas". Esta evidencia muestra la 


\section{Juan Salazar Parra}

intención del profesor de proteger o reforzar la imagen social del alumno e instarlo a colaborar.

Las estrategias de cortesía que apoyan el desarrollo de cada una de las tesis formuladas se orientan, en el caso de la tesis 1 , hacia la imagen negativa del destinatario, tratando de atenuar o minimizar los enunciados que podrían coaccionar su acción, tales como "¿Hay alguien que esté a favor de esa tesis o alguien que esté en contra?”; “No sé si te acuerdas”.

Con respecto a la tesis 2, el profesor intenta ser más positivo, empleando las estrategias de Satisfacción de los deseos del otro y apelando al Terreno Común, como es el caso de: "Y a ustedes les pareció muy mal”; “Cuando yo les dije ustedes reclamaron”; “¿Sí?”.

Finalmente, en la fase de cierre, ambas interacciones argumentativas se centran en un enfoque un tanto más cooperativo que cortés. Sin embargo, se plantean proposiciones tales como: "Siempre va a ser un tema que necesita de mucha dedicación” o: “Tú, de alguna manera, responsabilizas también a la sociedad, a la educación”.

Por su parte, los alumnos utilizan en mayor grado las estrategias de Cortesía encubierta (Ironía y Ambigüedad), lo que indica que en ellos no existe una única intención comunicativa: "No sé po’h, cualquier cosa menos una persona de bien común”; "Qué quieren, que vaya a meterse a secar platos”; “O sea, qué pretenden ¿Que la mamá sea una ignorante?”. Sin embargo, esta situación no ocurriría en la interacción "guiada”, lo que se debe a que la Cortesía encubierta supone, por un lado, confianza entre los interlocutores, situación que generaría sólo la interacción "espontánea”, al no estar subsumidos en roles psicosociales. Por otro lado, la interacción "guiada" supone una delimitación temática y una unívoca intención argumentativa, dada su preparación, lo que no ocurriría en la otra interacción, en que la intención de discutir no está planteada de antemano sino que se generaría en la interacción misma.

Por otra parte, en la secuencia “espontánea”, los alumnos tienden a favorecer el acuerdo que les permite, en general, conducir sin someter la discusión a una hostilidad latente entre los interlocutores. Algo similar ocurre en la interacción "guiada", en la cual los participantes asumen ciertos roles y emplean estrategias de cortesía con el fin de lograr adherir a sus puntos de vista, la mayor cantidad de posiciones divergentes.

A pesar del uso amplio de estrategias de Cortesía encubierta y del amplio favorecimiento del acuerdo, evitando amenazar la imagen pública del destinatario (Marinkovich, 2007a) los alumnos tienden a la informatividad, es decir, a enunciar sus argumentos de forma concisa, relevante y clara. 
Estrategias de cortesía verbal en interacciones argumentativas

\section{COMENTARIOS FINALES}

En las interacciones del profesor y de los alumnos, el sujeto que gestiona y negocia las intervenciones de los interlocutores es aquel que emplea el rol de moderador; a él se le atribuye la función del planteo de la(s) tesis y la consecución del diálogo. Los otros estudiantes participan exponiendo sus puntos de vista a favor o en contra de la(s) tesis formulada(s), ya sea defendiendo o atacando con argumentos sus posturas. La decisión de dar por terminada la discusión "guiada” la toma el moderador. Por otra parte, los enunciados, tanto de las tesis como de los argumentos son incompletos y, en ocasiones, ambiguos, y su expresión evidencia el devenir propio de la oralidad, plena de pausas, solapamientos, reiteraciones, vacilaciones, evanescencia y, también, empleo de cierto registro informal.

La participación de los alumnos en la interacción "espontánea” se centra en unos pocos, prueba de ello es que de 42 alumnos sólo 18 asumen participación en las diversas intervenciones, lo que explica el magro desarrollo, por ejemplo, de la tesis 1 . Situación opuesta es la que se aprecia en la interacción "guiada” en la que, por su propia naturaleza evaluativa, sólo intervienen los estudiantes, lo que permite desarrollar y dar por terminada, con mayor certeza, la tesis inicial. En esta modalidad los adolescentes justifican sus opiniones, consideran las de los demás, negocian los puntos de vista de los interlocutores y contra-argumentan para llegar a resolver el desacuerdo inicial (Cros, 2005).

En cuanto a las estrategias de cortesía que regulan la interacción, salvaguardando la imagen del emisor y no agrediendo la del destinatario, sobre todo en instancias controvertidas y poco reguladas (como la interacción "espontánea” o plenario) —en las que es preciso mantener una actitud cooperativa y conciliadora entre los participantes - es el profesor quien oficia como mediador, apoyado en las intervenciones de algunos alumnos, como es el caso de A8 y A10, quienes favorecen evidentemente las formas de acuerdo, pues permiten, en lo posible, conducir sin tensiones el desarrollo de la situación argumentativa en la secuencia analizada.

En la otra secuencia estudiada, los panelistas y el moderador, asumen roles psicosociales y emplean, aunque en menor grado, estrategias de cortesía con el fin de favorecer opiniones similares. Esto ocurre, de manera especial, entre aquellos panelistas que apoyan un mismo punto de vista. En este caso, los enunciados de cortesía se presentan en menor cantidad debido a la intención informativa y de lo prototípico de una discusión oral.

Muchos de los enunciados producidos por los interlocutores como, por ejemplo, cuando un alumno formula un punto de vista contrario al de un compañero, amenazan la imagen positiva del último. En este caso, el alumno 


\section{Juan Salazar Parra}

utiliza una estrategia de cortesía que atenúa dicha amenaza, presentando la divergencia como una conformidad parcial, mediante la adversatividad "pero" ("sí, pero...") o se puede acudir a la impersonalidad ("Actualmente se trata el tema en forma más relajada”, o "Hay quienes se hacen cargo de ellos”).

Las secuencias analizadas corresponden a dos modelos de argumentación como actividad discursiva interactiva, aunque con fines radicalmente opuestos. Mientras una interacción se ha planificado y preparado tanto en lo informativo como en su forma y propósito, la otra ha resultado de una discusión espontánea que formula sus tópicos en el tránsito de la discusión y puede ir cambiando de objetivos. A pesar de las diferencias, ambas interacciones se producen frente a una situación problemática (controversia, desacuerdo o polémica) que compromete a diferentes actores y que tiene la finalidad de proponer un punto de vista, defenderlo o atacarlo para que se resuelva dicha situación problemática.

Tal como se mencionara al comienzo, esta caracterización del discurso argumentativo oral corresponde a la descripción de la competencia argumentativa que, según MINEDUC, debieran manifestar los estudiantes de enseñanza media, lo anterior resulta fundamental, junto con la toma de posiciones opuestas con respecto a un punto de vista y a la actuación comunicativa en orden a dar razones a favor, o en contra, de tal punto de vista en un sentido coherente con sus posiciones y un mínimo de compromiso con éstas. Claro está que los estudiantes no manifiestan amplio conocimiento ni uso del tipo de estrategias estudiadas en esta ocasión. Tal vez, la actividad "clase" coacciona a los estudiantes, quienes se ven en la necesidad de responder a interrogantes declarativas, siempre concisas y cooperativas, en desmedro de la discusión crítica, abierta y cooperativa, propia del aprendizaje constructivo.

La enseñanza de esos últimos recursos y el desarrollo de actividades que propicien la discusión permitirían a los alumnos establecer mejores relaciones sociales, expandir sus conocimientos y posturas frente a los temas trascendentales de la vida y, en definitiva (Gómez, 2005), construir su propia historia a partir del lenguaje, como una de las vías más asequibles para lograr dichos propósitos. Por lo mismo, esta propuesta pretende seguir su desarrollo en áreas como la didáctica de la argumentación, planteando actividades y estableciendo patrones de conducta argumentativa con miras a potenciar la competencia argumentativa de los estudiantes e, incluso, del mismo docente.

Asimismo, la incorporación de las estrategias no verbales empleadas por los diferentes interlocutores y su relación o discordancia con el empleo de ciertas estrategias de cortesía, fortalece un estudio como éste. También, la sistematización de los roles prototípicos y la caracterización de los papeles 
argumentativos en una discusión, responden bien a la interrogante acerca de la competencia argumentativa de los alumnos en el contexto de aula.

\author{
Universidad Católica de Valparaíso* \\ Instituto de Literatura y Ciencias del Lenguaje \\ Av. Brasil 2830, Valparaíso (Chile) \\ juan.salazar.p@mail.ucv.cl
}

\title{
BIBLIOGRAFÍA
}

BAKER, Michael. “Argumentation and Constructive Interaction”, en Studies in Writing. Vol. 5. Foundations of Argumentative Text Processing. G. Rijlaarsdam \& E. Espéret (Series Eds.) \& Pierre Coirier and Jerry Andriessen (Vol. Eds.). Amsterdam: University of Amsterdam Press, 1999.

BARTH, Else \& KRABBE, Eric. From Axiom to Dialogue: A Philosophical Study of Logics and Argumentation. Berlin \& New York: Walter de Gruyter, 1982.

BROWN, Penelope \& LEVINSON, Stephen. Politeness. Some Universals in Language Use. Cambridge: Cambridge University Press, 1987.

CALSAMIGLIA, Helena \& TUSÓN, Amparo. Las cosas del decir. Barcelona: Ariel, 1999.

CROS, Anna. Convencer en Clase: Argumentación y Discurso Docente. Barcelona: Ariel Lingüística, 2003.

------- "La argumentación oral”, en El discurso oral formal. Contenidos de aprendizaje y secuencias didácticas. Monserrat Vilà i Santasusana (Coord.). Barcelona: Graó, 2005.

ESCANDELL, María Victoria. Introducción a la pragmática. Barcelona: Ariel, 1996.

GÓMEZ, Luis. "El lenguaje y el hombre”, en El hombre y su palabra. Marianne Peronard y Ximena Gómez (Eds.). Valparaíso: Ediciones Universitarias de Valparaíso, 2005.

GOFFMAN, Erving. Forms of talk. Londres: Basil Blackwell, 1981.

GRICE, Paul. "Logic and conversation”, en Syntax and semantics 3: Speech acts. New York: NY Academic Press, 1975.

MARINKOVICH, Juana. "La interacción argumentativa en el aula: fases de la argumentación y estrategias de cortesía verbal”, en Estudios en argumentación y retórica. Teorías contemporáneas y aplicaciones. Cristián Santibáñez y Bernardo Riffo (Eds.). Concepción: Universidad de Concepción, 2007a. 
------- "Las estrategias cognitivo-retóricas y la dimensión dialéctica de la argumentación oral en una clase de lengua castellana y comunicación”, en Revista Signos, Vol. 40, Nº. 63, 2007b.

MINEDUC. Lengua Castellana y Comunicación / Lenguaje y Comunicación. Programa de Estudio, Tercer Año Medio, Formación General. Educación Media. Santiago de Chile: Ministerio de Educación de Chile, 2004.

PERELMAN, Chain \& Olbrechts-Tyteca, Lucie. Traité de l"argumentation. La nouvelle rhétorique. París: PUF, 1958.

REYES, Graciela. El abecé de la pragmática. Madrid: Arco Libros, 1998.

TOULMIN, Stephen. The uses of argument. Cambridge: Cambridge University Press, 1958.

VAN EEMEREN, Franz \& GROOTENDORST, Robert. Argumentation, communication and fallacie. A pragmadialectical perspectiva. $\mathrm{N}$. Jersey: L. Erlbaum, 1992. 